\title{
Self-Assembly and Stability of Hydrogen-Bonded Networks of Bridged Triphenylamines on $\mathrm{Au}(111)$ and $\mathrm{Cu}(111)$
}

Christian Steiner, ${ }^{1}$ Bettina D. Gliemann, ${ }^{2[+]}$ Ute Meinhardt, ${ }^{2[+]}$ Martin Gurrath, ${ }^{3,4}$ Bernd Meyer, ${ }^{3,4}$ Milan Kivala, ${ }^{\star 2}$ Sabine Maier ${ }^{* 1,3}$

1 Department of Physics, Friedrich-Alexander University Erlangen-Nürnberg, Erwin-Rommel-Straße 1, 91058 Erlangen, Germany.

${ }^{2}$ Chair of Organic Chemistry I, Department of Chemistry and Pharmacy, Friedrich-Alexander University Erlangen-Nürnberg, Henkestraße 42, 91052 Erlangen, Germany.

${ }^{3}$ Interdisciplinary Center for Molecular Materials, Friedrich-Alexander University Erlangen-Nürnberg

${ }^{4}$ Computer-Chemistry-Center, Friedrich-Alexander University Erlangen-Nürnberg, Nägelsbachstraße 25, 91052 Erlangen, Germany

*milan.kivala@fau.de

* sabine.maier@fau.de

[+] These authors contributed equally to this work. 


\section{Temperature Dependence of CDTPA Networks on Au(111)}

While the ADTPA molecules formed extended islands already at RT, the CDTPA networks required moderate heating to $340 \mathrm{~K}$. Figure S1 shows the temperature evolution of CDTPA molecules deposited on $\mathrm{Au}(111)$ (a) at RT and (b) after subsequent annealing to $340 \mathrm{~K}$. The limited island size at RT is related to interstitial defects in the center of the pores (Figure S1c). The binding motif is the same at RT and $340 \mathrm{~K}$, but changes at $410 \mathrm{~K}$ (see Figure 5).
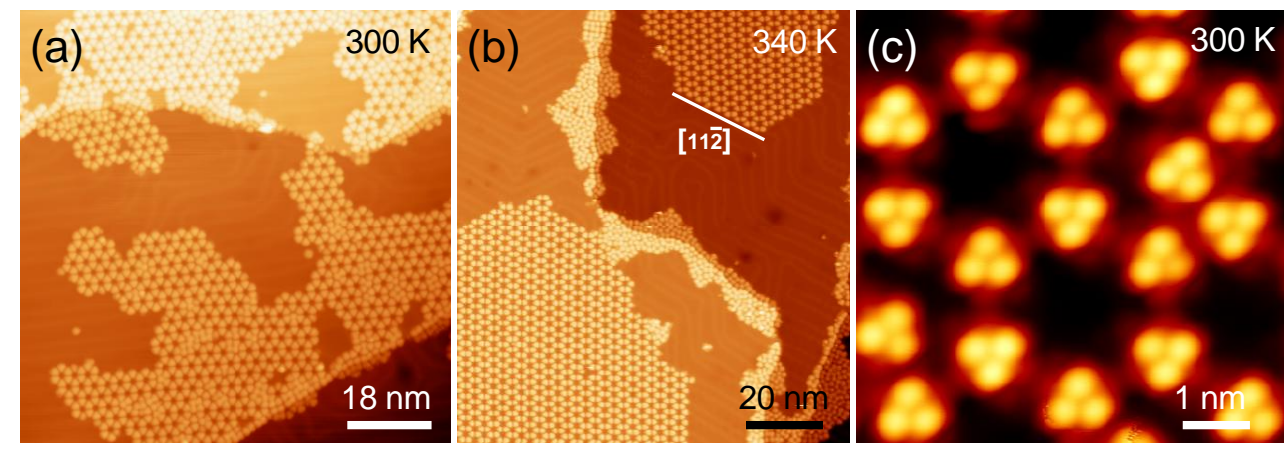

Figure S1. STM images of CDTPA (a) deposited at RT and (b) annealed to $340 \mathrm{~K}$. (c) Shows an interstitial defect in the honeycomb network deposited at RT. STM parameters: (a) $U=-2 \mathrm{~V}, I=100 \mathrm{pA}$, (b) $U=-100 \mathrm{mV}, I=40 \mathrm{pA}$, and (c) $U=-100 \mathrm{mV}, I=490 \mathrm{pA}$.

\section{Herringbone Reconstruction after Molecular Sublimation on Au(111)}

Figure S2 shows additional STM images of the (a) CDTPA and (b) ADTPA honeycomb network. The modulation in the apparent STM height is attributed to the underlying herringbone reconstruction of $A u(111)$. The Au herringbone reconstruction is unperturbed underneath the molecular network. The apparent height of the molecular layers in the STM images is around 3-4 $\AA$.
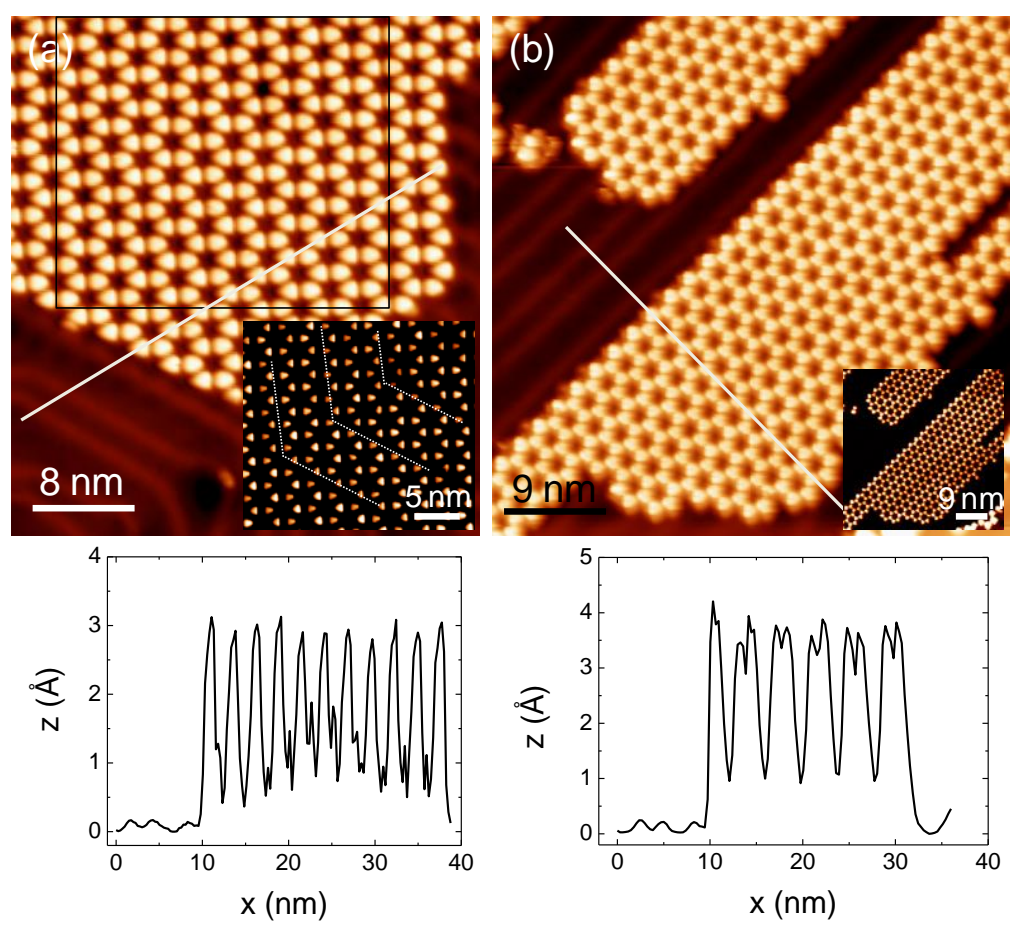

Figure S2. STM images with corresponding height sections of the (a) CDTPA and (b) ADTPA networks on Au(111) showing that the herringbone reconstruction remains unperturbed underneath the molecular network. STM parameters: (a) $U=-100 \mathrm{mV}, I=40 \mathrm{pA}$ and (b) $T=4.8 \mathrm{~K}, U=-2 \mathrm{~V}, I=50 \mathrm{pA}$. 


\section{Hydrogen-Bonding Motifs of the ADTPA Molecule}

DAT head groups provide multiple possibilities to form an ADTPA dimer using hydrogen bonds. The three common hydrogen-bonding motifs of the DAT group are the: (i) tip-to-tip, (ii) side-to-side, and (iii) tip-to-side configuration (see Figure 1). ${ }^{1}$ All three bonding motifs lead to a honeycomb network (see Figure S3 for tentative models based on the planar DFT optimized dimers). Each of the three bonding motifs results in a specific pore configuration, which can be unambiguously distinguished by the molecule-molecule distance and the orientation of the triangle, defined by the protrusions of the dimethylmethylene side groups (depicted as green triangles in Figure S3). On Au(111), exclusively the pore configuration with the tip-to-side bonding motif was observed (Figure S3c and d).

(a)

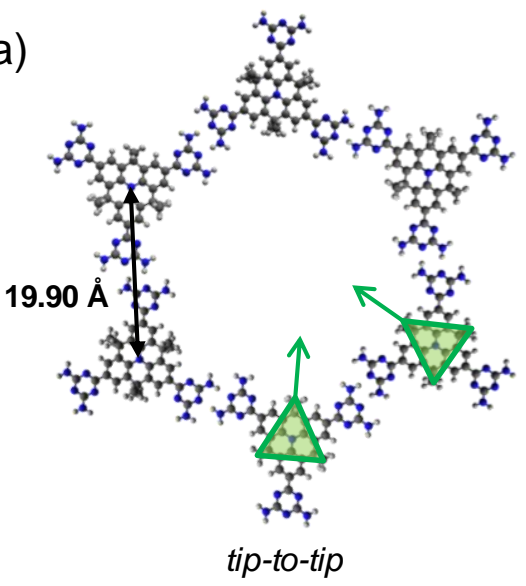

(c)

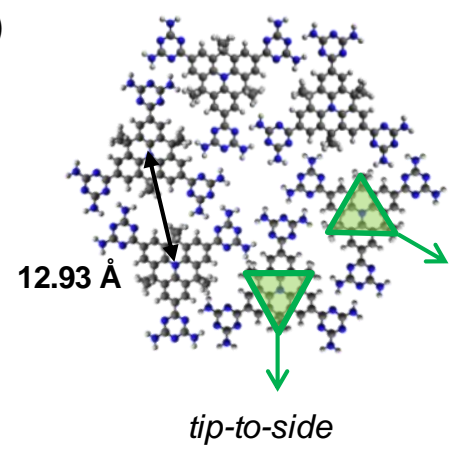

(b)

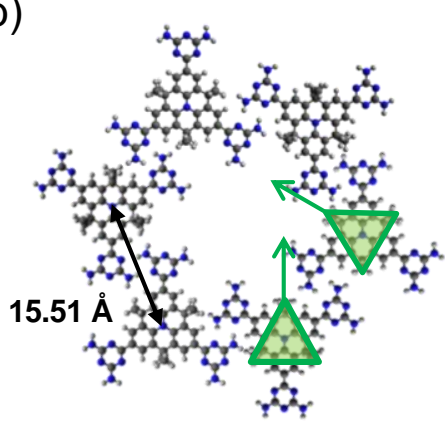

side-to-side

(d)

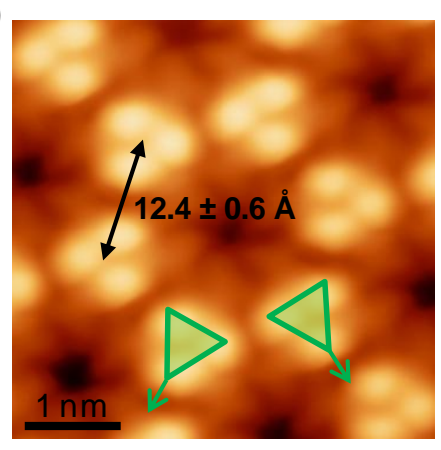

Figure S3. Planar honeycomb networks of the ADTPA molecule. The molecules are connected through the characteristic H-bonding motifs of the DAT groups: (a) Tip-to-tip, (b) side-to-side, and (c) tip-to-side. The unit-cells of the networks are calculated by DFT (PBE+D3). The triangles defined by the protrusions of the dimethyl side groups (depicted as green triangles) point to the center of the pore for (a) and (b) whereas they point out of the pore for the tip-to-side motif. The geometry of the tip-to-side networks agrees well with the networks observed in STM measurements (d). STM parameters: $U=-800 \mathrm{mV}, I=150 \mathrm{pA}$.

Figure S4 shows the structure of the DFT (PBE+D3) optimized ADTPA dimers in the planar (local energy minimum) and non-planar configuration (global energy minimum). In case of the planar geometries, we obtain a $N_{\text {triazine }} \cdots N_{\text {amino }}$ hydrogen bond length between $2.93 \AA$ and $3.03 \AA$ for all three bonding motifs. These values are in good agreement with calculated bond length of melamine ${ }^{2}(2.89 \AA-3.05 \AA)$ and lander-DAT molecules ${ }^{3}(2.89 \AA-2.92 \AA)$. The bond angle of $\mathrm{N}_{\text {triazine }} \cdots \mathrm{H}$ $\mathrm{N}_{\text {amino }}$ bonds in the planar ADTPA dimers is between $173^{\circ}$ and $178^{\circ}$, which is consistent with the calculations for melamine. ${ }^{2}$ For the tip-to-side and side-to-side motif, the planar configuration has a negligible out-of-plane rotation angle of the DAT group, i.e. the DAT group is mainly in-plane with the molecular scaffold. In contrast, in the non-planar configuration the DAT group rotates by approximately $15^{\circ}$ with respect to the plane of the molecular scaffold. Except for the non-planar tip-to-side motif, all amino groups are planar when participating in $\mathrm{H}$-bonds (see encircled zooms in Figure S4), and non-planar when 
not participating in hydrogen bonds (see encircled zooms in Figure S4). This means that the molecule-molecule interaction through the hydrogen bonds planarizes the amino group.

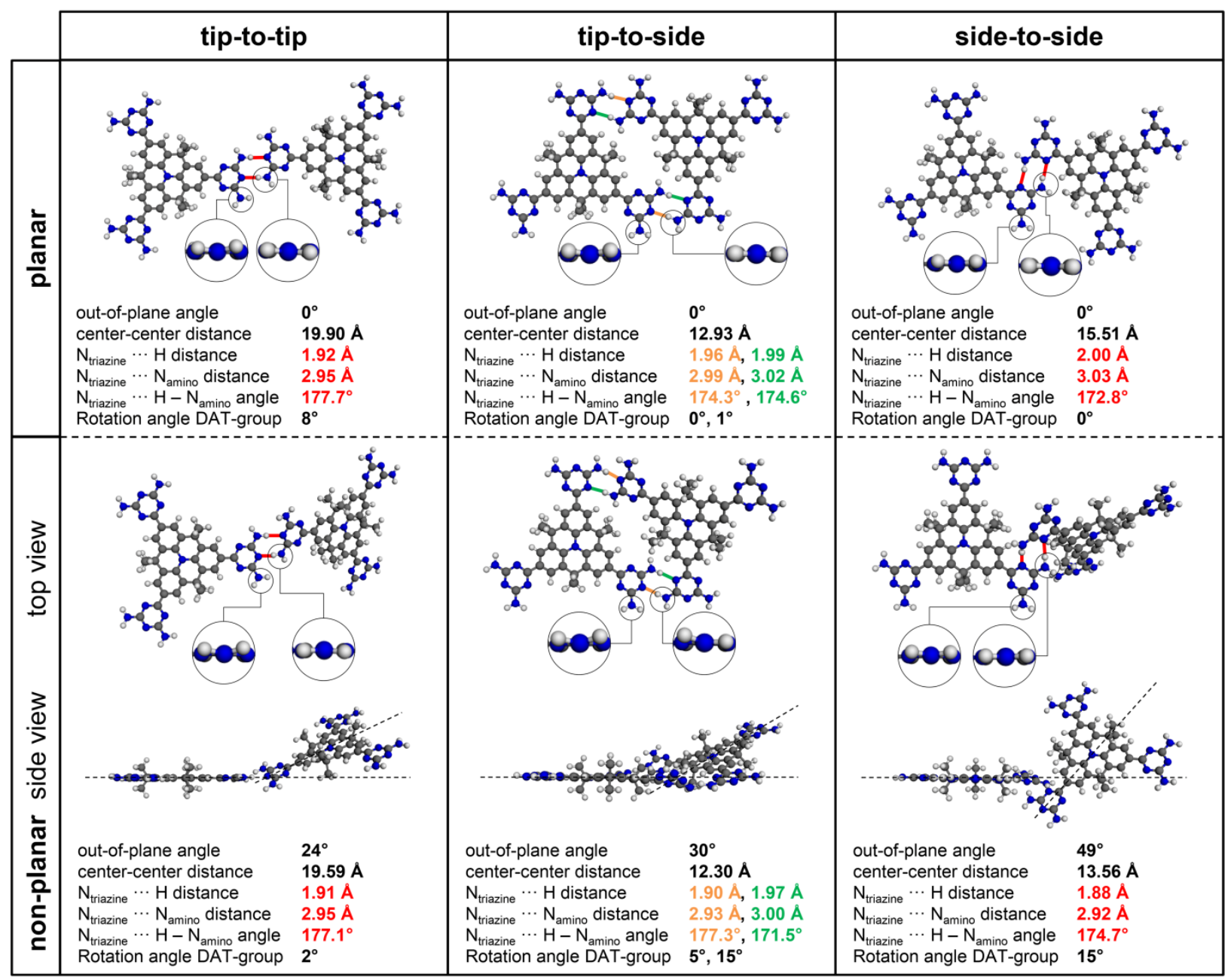

Figure S4. Structure of DFT (PBE+D3) optimized ADTPA dimers in the planar (local energy minimum) and non-planar (global energy minimum) conformation. The out-of-plane angle is indicated by dashed lines in the side view. The center-to-center distance corresponds to the distance between the central nitrogen atoms of neighboring molecules. The hydrogen bond distance is indicated by the colored lines. The rotation angle of the DAT groups is defined as the angle between the plane of the molecular scaffold and the plane of the DAT group. The encircled figures are zooms on amino groups which participate in hydrogen bonds and on amino groups which do not participate in hydrogen bonds. 


\section{Organizational Chirality of the ADTPA Molecule on Au(111)}

The ADTPA molecules are achiral. The tip-to-side bonding motif of the DAT head group offers two possibilities to form a dimer, which leads to an organizational chirality ${ }^{4}$ in the honeycomb motif. Homo-chiral domains, one with left-handed chirality and one with right-handed chirality, are shown in Figure S5. The chirality of the ADTPA network can be recognized by the $\pm 14.7^{\circ}$ angle between the molecular axis and the connection between two opposing $\mathrm{N}$-centers in a pore.
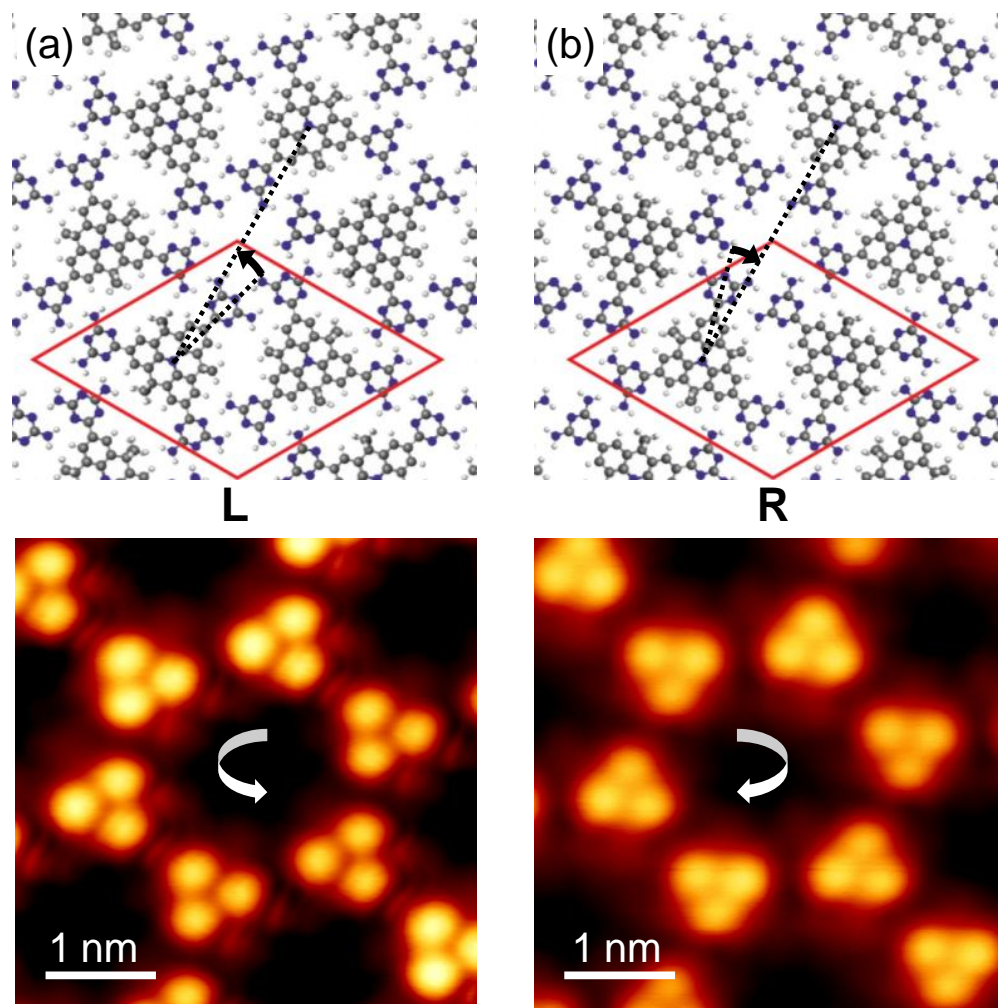

Figure S5. Organizational chirality in the ADTPA network on $\mathrm{Au}(111)$ : Structure of both homo-chiral domains with a corresponding STM image. (a) Left-handed chirality and (b) right-handed chirality. STM parameters: (a) $U=-850 \mathrm{mV}, I=550 \mathrm{pA}$; (b) $U=-300 \mathrm{mV}, I=550 \mathrm{pA}$.

\section{Deprotonation of CDTPA Molecules on Au(111)}

Upon annealing to $410 \mathrm{~K}$, most of the CDTPA molecules align in linear structures along the herringbone reconstruction on $\mathrm{Au}(111)$. Figure S6a outlines the relative orientation of the CDTPA molecules in the linear chains (410 K, green triangles) versus the honeycomb network (RT, blue triangles in the inset). The intermolecular distance along a single chain is $11.2 \pm 0.6 \AA$, and the offset between the molecular centers perpendicular to the chain direction is $1.22 \pm 0.06 \AA$ as obtained experimentally from the STM measurement. A tentative adsorption model of a CDTPA chain is presented in Figure S6b, where every second molecule (i.e. the equally oriented molecules) is separated by nine times the atomic distance along $\langle 11 \overline{2}\rangle\left(\sqrt{3} / 2 \cdot a_{111}\right.$ with $\left.a_{111}=2.88 \AA\right)$. Hence, the intermolecular distance along the chain axis is $11.22 \AA$ in the tentative model, which is in good agreement with the measurement. The offset perpendicular to the chain axis is $1 / 2 \cdot a_{111}=1.44 \AA$ in order to achieve an epitaxial fit of the chain to the atomic lattice, which is in the same order as the measured value. We attribute the altered self-assembly to a partial deprotonation of one carboxyl group, which can adsorb commensurate to the $\mathrm{Au}(111)$ lattice. We suggest that the molecules bind via $\mathrm{H}$-bonds between the intact carboxyl groups and the dimethyl groups. In addition, a STM image of a double chain is provided in Figure S6c. The triangles in the lower part of the figure depict the different orientations of the molecules, which are the same relative orientations as observed in the linear chain indicating the same bonding along the chain axis. 

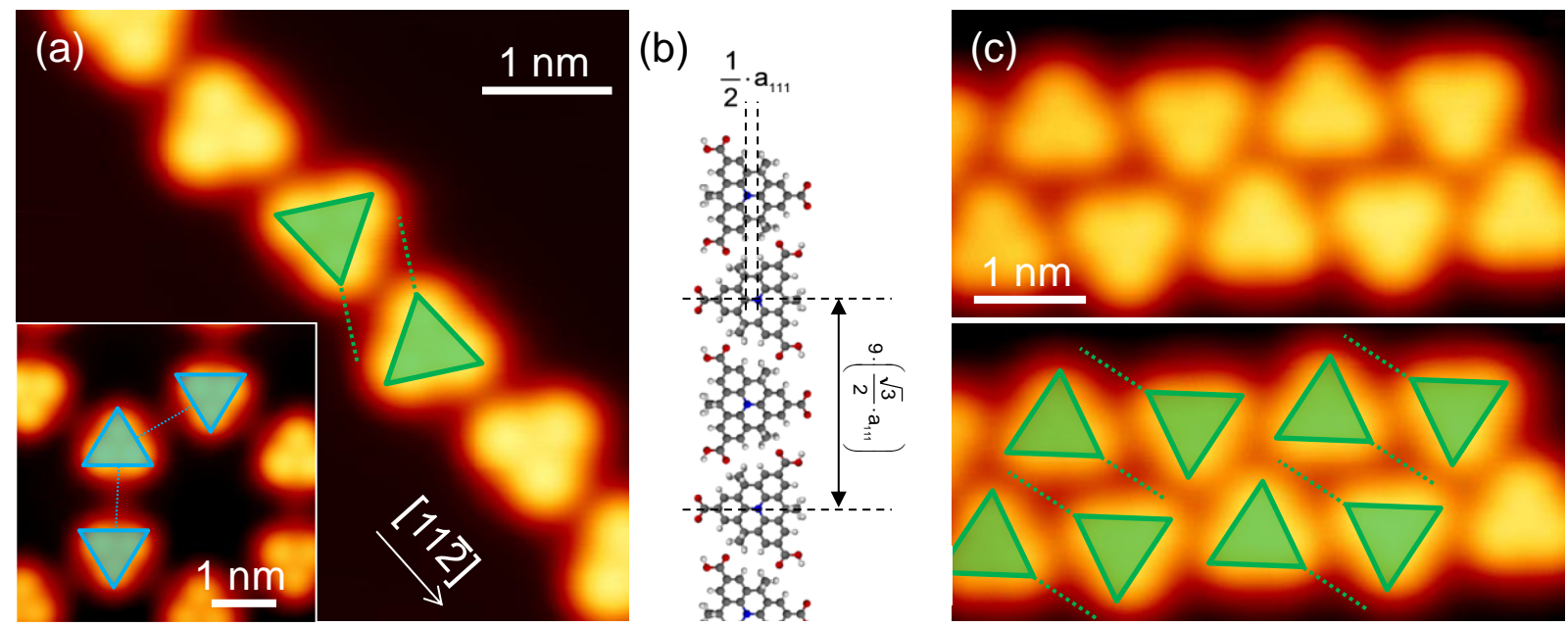

Figure S6. Linear arrangement of CDTPA after annealing to $410 \mathrm{~K}$ on $\mathrm{Au}(111)$. (a) Linear chain of CDTPA molecules. The green triangles highlight the relative orientation between neighboring molecules, which differs from the honeycomb network (blue triangles, inset) before heating. (b) Tentative model for the linear chains based on geometrical reasoning: The distances between the molecules are a multiple of Au lattice constants, yielding a distance between equally oriented molecules of twice $11.22 \AA$, which is 9 times $\sqrt{3} / 2 \mathrm{a}_{111}$. (c) Zoom image on a double row with the same relative orientation of the molecules as in the single chains. STM parameters: $T=4.6 \mathrm{~K}$; (a) $U=-500 \mathrm{mV}$, $I=150 \mathrm{pA}$; inset: $U=-80 \mathrm{mV}, I=1.5 \mathrm{nA}$; (c) $U=-4 \mathrm{~V}, I=10 \mathrm{pA}$.

\section{Cleavage of Methyl Groups upon Annealing}

A reduction of the apparent height is observed in STM measurements for ADTPA molecules annealed to $520 \mathrm{~K}$ on $\mathrm{Cu}(111)$. In Figure S7 two apparent heights of the molecules can be distinguished. The dimmer molecules, which are in the majority, have an apparent height of $0.93 \pm 0.06 \AA$, while a few molecules appear brighter corresponding to a height of $2.20 \pm 0.19 \AA$. In measurements without surface annealing, the apparent height of the intact ADTPAs on $\mathrm{Cu}(111)$ is around $2.2 \AA$. We attribute the height reduction to the cleavage of the methyl groups of ADTPA. A similar reduction of the apparent height by about $52 \%$ was described by Bieri et al. after annealing dimethylmethylene bridged triphenylamines on $\mathrm{Ag}(111)$, which was also explained with the cleavage of the dimethyl groups. ${ }^{5}$
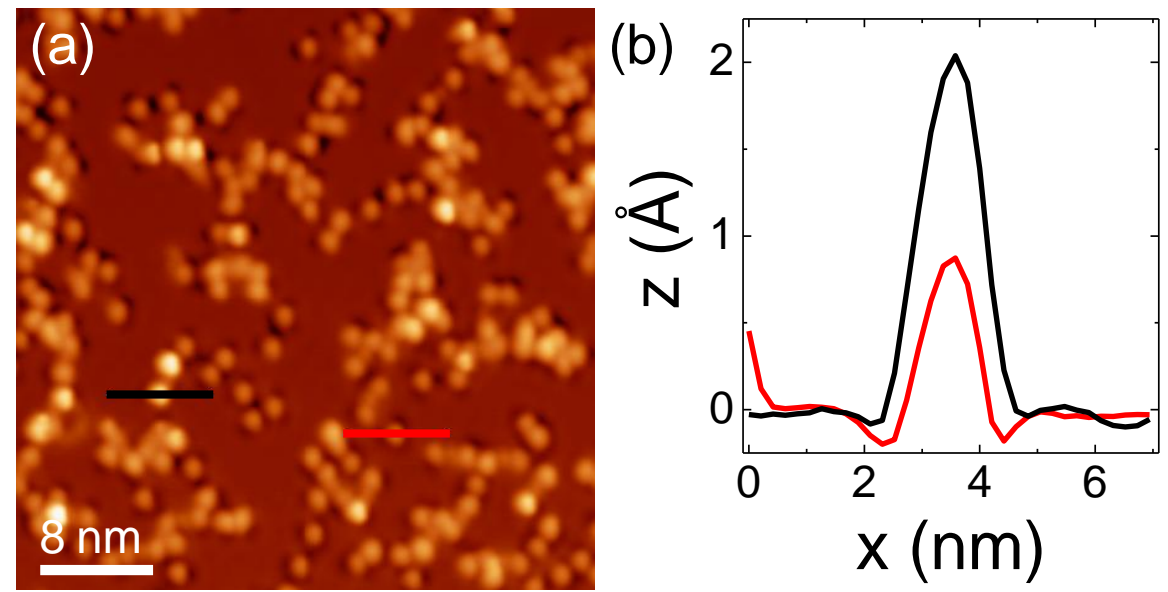

Figure S7. ADTPA molecules evaporated on $\mathrm{Cu}(111)$ and annealed to $520 \mathrm{~K}$. The molecules exhibit two distinct heights; The dimmer molecules have an approximate height of $0.9 \AA$, while the brighter have an approximate height of $2.2 \AA$. This height difference can be attributed to the cleavage of methyl groups. STM parameters: $T=4.8 \mathrm{~K}, U=-1 \mathrm{~V}, I=20 \mathrm{pA}$. 


\section{Commensurability of ADTPA Molecules on $\mathrm{Cu}(111)$}

Figure S8 shows a tentative model of the (a) $\alpha$ - and (b) $\beta$-phase. The intermolecular distances and angles correspond to the calculated gas phase model (GP). The orientation of the dimers with respect to the $\mathrm{Cu}(111)$ substrate is extracted from the STM measurements. The red box highlights the unit cell of a potentially commensurable assembly (C). Despite the difference between the GP unit cell and the $\mathrm{C}$ unit cell, three $\mathrm{N}_{\text {aminos }}$ per molecule adsorb near $\mathrm{Cu}$ atop sites (highlighted in red). The difference in length between the GP unit cell and the $\mathrm{C}$ unit cell is less than $3 \%$, and the difference in angles between the two unit cell vectors is less than $2^{\circ}$. A possible commensurate self-assembly of the $\alpha$ - and $\beta$-phase is described by the adsorption matrices

$$
\left(\begin{array}{cc}
9 & 0 \\
5 & 10
\end{array}\right) \quad \text { and } \quad\left(\begin{array}{cc}
10 & -4 \\
5 & 10
\end{array}\right)
$$

respectively (see Figure S8).
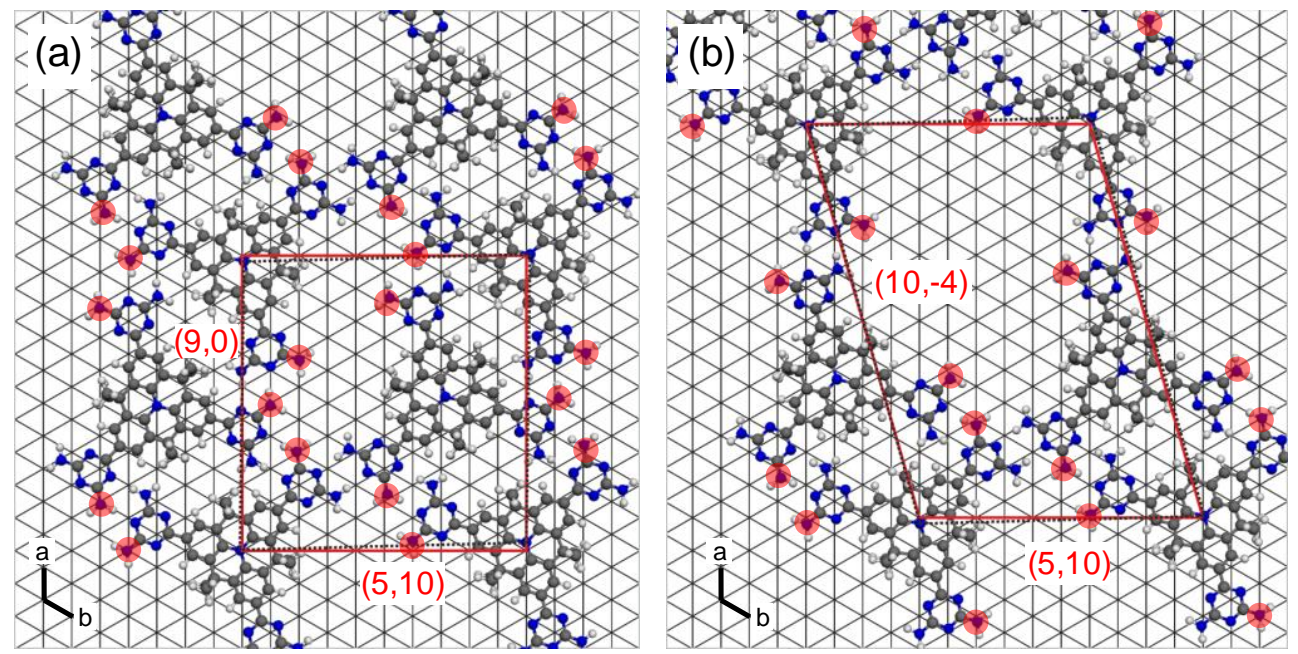

Figure S8. Tentative models, based on the DFT-optimized dimers, of the (a) $\alpha$ - and (b) $\beta$-phase of ADTPA superimposed on a Cu(111) atomic lattice according to the orientations observed in STM measurements. The unit cell of the gas phase model is depicted as dashed black box, while the red box highlights a unit cell which is commensurable to the underlying lattice. Red circles highlight three $\mathrm{N}_{\text {amino }}$ per molecule which adsorb near Cu atop sites.

Further, we estimated the coincidence misfit ${ }^{6}$ between the three dimers and the underlying Cu lattice, $\delta=\left(\mid \mathbf{a}_{s} /-a_{f}\right) /\left(\mid \mathbf{a}_{s} /\right)$, where $\mathbf{a}_{s}$ denotes the lattice vector closest to the dimer axis (i.e. intermolecular center-to-center distance) and $a_{f}$ stands for the molecular center-to-center distances taken from the DFT-optimized (PBE+D3) dimers (see Figure S4). Figure S9 shows the calculated gas phase dimers of the (a) tip-to-side, (b) tip-to-tip, and (c) side-to-side motif on-top of a Cu(111) lattice, such that one molecule is nearly commensurate to the $\mathrm{Cu}$ lattice. The length of $\mathbf{a}_{\mathrm{s}}$ is $\sqrt{27} \mathbf{a}$ (tip-to-side), $\sqrt{57} \mathbf{a}$ (tip-to-tip), and $\sqrt{31}$ a (side-to-side), respectively, with the lattice parameter $a=2.556 \AA$ on the $\mathrm{Cu}(111)$ surface (see green arrows in Figure S9). Hence, we obtain for the misfit $\delta=2.6 \%$ (tip-to-side, $\delta=2.9 \%$ for the monolayer), $\delta=-3.1 \%$ (tip-to-tip), and $\delta=-9.0 \%$ (side-to-side), respectively. The misfit is highest for the side-to-side motif, which may explain the fact that it is not observed in our STM measurements. In contrast, both tip-to-side and tip-to-tip motifs have misfits, which are low enough to be reasoned with arching of the molecule ${ }^{7}$, or a certain degree of freedom in rotation ${ }^{3}$ and tilt $^{8}$ of the DAT groups. 

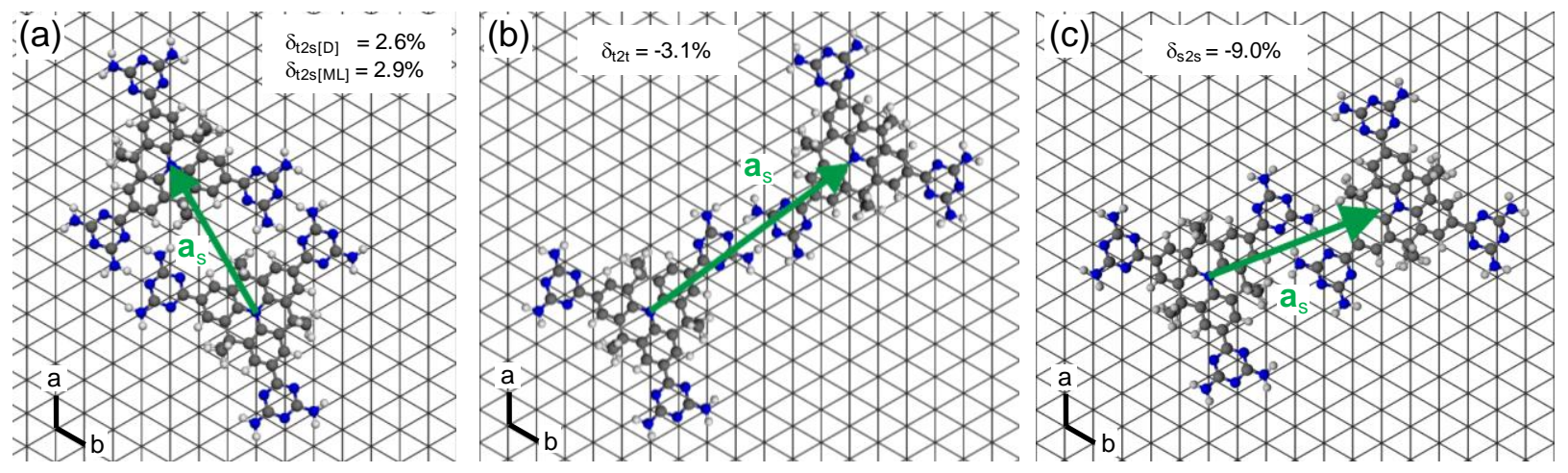

Figure S9. DFT-optimized ADTPA dimers with the three possible bonding motifs which are aligned with respect to the lattice according to the orientations of the molecules extracted from STM measurements. The green arrows highlight the lattice vector which coincides best with the dimer axis (i.e. the connection line between the central $\mathrm{N}$ atoms). Along these vectors the misfit is calculated.

\section{Deprotonation of ADTPA Molecules on $\mathrm{Cu}(111)$}

The strong interaction of ADTPA molecules with the $\mathrm{Cu}(111)$ surface is manifested in the particular orientation of each molecule (dashed black lines in Figure S10) with respect to the underlying Cu substrate (red lines in Figure S10). The ordered $\alpha$ - and $\beta$ - phase show three rotational domains and the organizational chirality provides two configurations for the dimer. Therefore, in total six orientations can be observed for the monomer at RT (Figure S10a). For the partially dissociated dimer the orientations are the same as for intact one at RT (Figure S10b). The partially deprotonated monomer with at least one depression at previous DAT group sites is oriented in three directions, i.e. perpendicular to the lattice directions (Figure S10c). The same orientation is observed for the partially deprotonated dimer connected through metal-ligand bonds (Figure S10d).
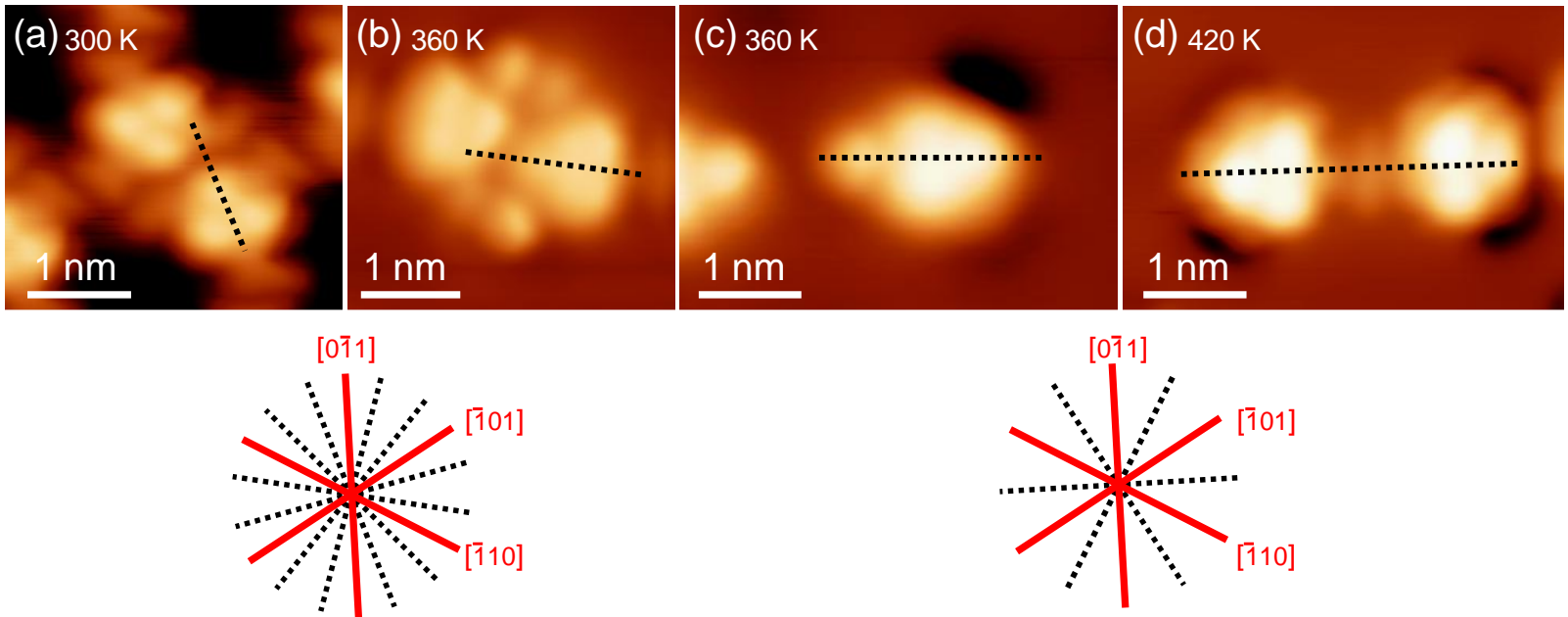

Figure S10. Orientation of intact and partially deprotonated CDTPA molecules: (a) Intact hydrogen-bonded dimer, (b) partially deprotonated hydrogen-bonded dimer, (c) partially deprotonated monomer and (d) deprotonated dimer interacting through metal-ligand bonds. The orientations of the molecular axes are schematically represented by black dashed lines, and the lattice orientations of the underlying $\mathrm{Cu}(111)$ surface are drawn in red in order to depict the relative orientation of the molecules with respect to the lattice. STM parameters: (a) $U=-1.9 \mathrm{~V}$, $I=610 \mathrm{pA}$; (b) $U=-0.35 \mathrm{~V}, I=300 \mathrm{pA}$; (c) $U=-0.25 \mathrm{~V}, I=600 \mathrm{pA}$; (d) $T=4.7 \mathrm{~K}, U=-0.55 \mathrm{~V}, I=510 \mathrm{pA}$. 


\section{References}

(1) Maly, K. E.; Gagnon, E.; Maris, T.; Wuest, J. D. Engineering Hydrogen-Bonded Molecular Crystals Built from Derivatives of Hexaphenylbenzene and Related Compounds. J. Am. Chem. Soc. 2007, 129, 4306-4322.

(2) Mura, M.; Martsinovich, N.; Kantorovich, L. Theoretical Study of Melamine Superstructures and Their Interaction with the $\mathrm{Au}(111)$ Surface. Nanotechnology 2008, 19, 465704.

(3) Yu, M.; Kalashnyk, N.; Xu, W.; Barattin, R.; Benjalal, Y.; Lægsgaard, E.; Stensgaard, I.; Hliwa, M.; Bouju, X.; Gourdon, A., et al. Supramolecular Architectures on Surfaces Formed through Hydrogen Bonding Optimized in Three Dimensions. ACS Nano 2010, 4, 4097-4109.

(4) Barlow, S. M.; Raval, R. Complex Organic Molecules at Metal Surfaces: Bonding, Organisation and Chirality. Surf. Sci. Rep. 2003, 50, 201-341.

(5) Bieri, M.; Blankenburg, S.; Kivala, M.; Pignedoli, C. A.; Ruffieux, P.; Müllen, K.; Fasel, R. Surface-Supported 2D Heterotriangulene Polymers. Chem. Commun. 2011, 47, 10239-10241.

(6) Forrest, S. R. Ultrathin Organic Films Grown by Organic Molecular Beam Deposition and Related Techniques. Chem. Rev. 1997, 97, 1793-1896.

(7) Gottardi, S.; Müller, K.; Moreno-López, J. C.; Yildirim, H.; Meinhardt, U.; Kivala, M.; Kara, A.; Stöhr, M. Cyano-Functionalized Triarylamines on $\mathrm{Au}(111)$ : Competing Intermolecular Versus Molecule/Substrate Interactions. Adv. Mater. Interfaces 2014, 1, 1300025.

(8) Cañas-Ventura, M. E.; Ait-Mansour, K.; Ruffieux, P.; Rieger, R.; Müllen, K.; Brune, H.; Fasel, R. Complex Interplay and Hierarchy of Interactions in Two-Dimensional Supramolecular Assemblies. ACS Nano 2011, 5, 457-469. 